DOI https://doi.org/10.30525/978-9934-26-019-3-6

\title{
THE STIFFNESS MATRIX OF INFINITE HEXAHEDRAL FINITE ELEMENT FOR FIBER COMPOSITE MATERIAL BASED ON THE MOMENT SCHEME
}

\section{Manko N. I.-V., Spytsia O. G.}

\section{INTRODUCTION}

The unique combination of mechanical properties of composite materials allows them to find new applications in various industries: energy, aerospace, construction, transportation, etc. So, one of the branches where they are used is the use of composite materials in the creation of conveyor belts, escalators, conveyor belts, sports and transport road surfaces.

The determination of the stress-strain state of such construction faces a number of mathematical and computational difficulties. Thus, the presence of composite material, which is heterogeneous in its structure, complicates the mathematical model of construction, which must take into account the presence of a large number of reinforcing elements. In addition, the described construction consists of elements with a specific geometric shape and sizes. These are usually confined strips or very long lengths that can be considered infinite. In the course of operation, such constructions test the load in the local area, which is considerably inferior in sizes to the length of the construction. Therefore, infinite-size modeling is an important problem in solving practical problems.

The finite element method was applied for research of infinite areas in one-dimensional and two-dimensional formulations. Application of infinite finite element with use of ANSYS for solving dynamic problem is shown in one- and two-dimensional cases [1]. Improving of calculation accuracy by use of special approximating functions for infinite finite element is proposed in the article [2]. Research of infinite two-dimensional medium with infinite finite element is described in the monography [3]. In the article [4] solving of static problems for infinite areas through infinite finite elements is represented. Analysis of underground excavations for unlimited areas based on infinite element method is shown in [5]. Construction of the stiffness matrix of infinite 
finite element for isotropic weak elastic material is developed in threedimensional case [6].

\section{Methods}

To model an infinite environment in one direction from a fibrous composite material, we use a hexagonal spatial finite element.

The origin of the coordinate system of a finite element we place in the center of the cube, and the axis we arrange so that they are parallel to the edges of the finite element and the $x_{3}$ axis would correspond to an infinite direction (Fig. 1).

We introduce two local coordinate systems: a local finite element coordinate system and a local reinforcement system. Reinforcement is performed in the $x_{2} O x_{3}$ plane. The coordinate system of finite element $O x_{1} x_{2} x_{3}$ and the coordinate system of reinforcement $O x_{1}^{\prime \prime} x_{2}^{\prime \prime} x_{3}$ are connected with the angle $\alpha$, which determines the fiber stacking direction relative to the axis $x_{2}$ (Fig. 1).

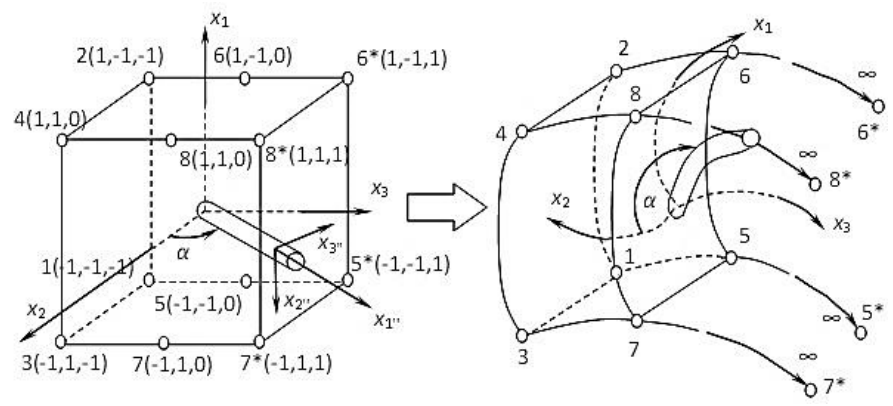

Fig. 1. The "infinite" finite element for fiber composite material

Using special approximation functions, we model infinity in the global Cartesian coordinate system $\left(O z_{1}^{\prime} z_{2}^{\prime} z_{3}^{\prime}\right)$. We select special approximating functions so that the nodes $5^{*}, 6^{*}, 7^{*}, 8^{*}$ are displayed on the infinity in the direction of the axis $z_{3}^{\prime}$.

We construct the stiffness matrix of the "infinite" finite element on the basis of the variational principle of Lagrange:

$$
\delta \tilde{\Pi}=\delta \tilde{W}-\delta \tilde{A},
$$


where $\tilde{\Pi}-$ the potential energy of the construction, $\tilde{W}$ - the energy of elastic deformation of the construction, $\tilde{A}-$ the work of external forces.

We consider the variation of the energy of elastic deformation of a single finite element in a matrix form:

$$
\delta W=\iiint_{V}\left\{\delta \varepsilon_{i j}\right\}^{T}\left[C^{i j k l}\right]\left\{\varepsilon_{k l}\right\} d V
$$

where $\left\{\varepsilon_{k l}\right\}=\left\{\varepsilon_{11}, \varepsilon_{12}, \varepsilon_{13}, \varepsilon_{21}, \varepsilon_{22}, \varepsilon_{23}, \varepsilon_{31}, \varepsilon_{32}, \varepsilon_{33}\right\}^{T}$ - the deformation vector, $\left\lfloor C^{i j k l}\right\rfloor$ - the matrix of elastic constants of material in the coordinate system of a finite element $x_{i}$

In the coordinate system of reinforcement, the composite material is homogeneous transtropic. The effective elastic properties of the transtropic material are determined by five mechanical characteristics: $E_{1}, E_{2}$ - the elastic modules (longitudinal and transverse, respectively), $G_{12}, G_{23}$ - the shear modules, and $v_{12}$ - the Poisson coefficient. Here, the first direction is determined by the direction of reinforcement of fiber, and the second and the third determine the plane of isotropy of the material. Other elastic constants are defined as follows:

$$
\begin{gathered}
v_{21}=v_{12} \frac{E_{2}}{E_{1}}, v_{23}=\frac{E_{2}}{2 G_{23}}-1, \\
v_{13}=v_{12}, v_{31}=v_{21}, v_{32}=v_{23}, \\
E_{3}=E_{2}, G_{13}=G_{12} .
\end{gathered}
$$

The components of the tensor of elastic constants $C^{i^{i j^{\prime \prime}} k^{\prime \prime} l^{\prime \prime}}$ for the transtropic environment in the coordinate system of reinforcement are determined by the following formulas [7]:

$$
\begin{aligned}
& C^{1^{\prime \prime} 1^{11} 1^{\prime \prime}}=\frac{E_{1}}{\Delta^{*}}\left(1-v_{23} v_{32}\right), C^{1^{\prime \prime} 1^{\prime \prime 2} 2^{\prime \prime}}=\frac{E_{2}}{\Delta^{*}}\left(v_{12}+v_{13} v_{32}\right), \\
& C^{1^{11} 1^{\prime \prime} 3^{\prime \prime}}=\frac{E_{3}}{\Delta^{*}}\left(v_{13}+v_{12} v_{23}\right), C^{2^{\prime 2} 2^{\prime \prime} 1^{\prime \prime}}=\frac{E_{1}}{\Delta^{*}}\left(v_{21}+v_{31} v_{23}\right), \\
& C^{2^{\prime \prime} 2^{\prime \prime} 2^{\prime \prime}}=\frac{E_{2}}{\Delta^{*}}\left(1-v_{13} v_{31}\right), C^{2^{2} 2^{\prime \prime} 3^{\prime \prime} 3^{\prime \prime}}=\frac{E_{3}}{\Delta^{*}}\left(v_{23}+v_{21} v_{13}\right),
\end{aligned}
$$




$$
\begin{gathered}
C^{3^{\prime \prime} 3^{\prime \prime} 1^{\prime \prime} 1^{\prime \prime}}=\frac{E_{1}}{\Delta^{*}}\left(v_{31}+v_{21} v_{32}\right), C^{3^{\prime \prime} 3^{\prime \prime} 2^{\prime \prime} 2^{\prime \prime}}=\frac{E_{2}}{\Delta^{*}}\left(v_{32}+v_{31} v_{12}\right), \\
C^{3^{\prime \prime} 3^{\prime \prime} 3^{\prime \prime}}=\frac{E_{3}}{\Delta^{*}}\left(1-v_{21} v_{12}\right), \\
C^{1^{\prime \prime} 2^{\prime \prime} 1^{\prime \prime}}=\frac{G_{12}}{2}, C^{1^{\prime \prime} 3^{\prime \prime} 1^{\prime \prime}}=\frac{G_{13}}{2}, C^{2^{\prime \prime} 3^{\prime \prime} 2^{\prime \prime}}=\frac{G_{23}}{2}, \\
\Delta^{*}=\left(1-v_{23} v_{32}-v_{12} v_{21}-v_{12} v_{23} v_{31}-v_{13} v_{21} v_{32}-v_{13} v_{31}\right),
\end{gathered}
$$

where $E_{1}, E_{2}, E_{3}$ - the elastic modules in the respective directions of the reinforcement system, $G_{i j}$ - the shear modules of the transtropic material, $v_{i j}$ - the Poisson coefficients.

In solving spatial problems, they use effective elastic constants obtained from different theories. One of the simplest and most common groups of formulas is the mixtures rule [8]:

$$
\begin{gathered}
E_{1}=E_{c} f+E_{m}(1-f), \\
E_{2}=\frac{E_{c} E_{m}}{E_{m} f+E_{c}(1-f)}, \\
G_{12}=\frac{G_{c} G_{m}}{G_{c}(1-f)+G_{m} f}, \\
G_{12}=\frac{G_{c} G_{m}}{G_{c}(1-f)+G_{m} f}, \\
G_{23}=\frac{\chi_{m}+f+(1-f) \frac{G_{m}}{G_{c}}}{(1-f) \chi_{m}+\left(1+f \chi_{m}\right) \frac{G_{m}}{G_{c}}} G_{m}, \\
v_{12}=v_{c} f+v_{m}(1-f),
\end{gathered}
$$

where $\chi_{m}=3-4 v_{m}, f-$ the volumetric fiber content, $E_{c}, G_{c}, v_{c}-$ the elastic modulus, the shear modulus and the Poisson coefficient of the fiber material, $E_{m}, G_{m}, v_{m}$ - the elastic modulus, the shear modulus and the Poisson coefficient of the matrix material. 
Another group of formulas was obtained by Abolin'sh D.S. [9]:

$$
\begin{gathered}
E_{1}=E_{c} f+E_{m}(1-f), \\
E_{2}=\frac{e E_{1}}{(f+e(1-f))((1-f)+e f)-f(1-f)\left(e v_{m}-v_{c}\right)^{2}}, \\
G_{12}=\frac{1-f+g(f+1)}{f+1+g(1-f)} G_{m}, \\
G_{23}=\frac{g}{f+g(1-f)} G_{m}, \\
v_{12}=v_{c} f+v_{m}(1-f),
\end{gathered}
$$

where $e=\frac{E_{c}}{E_{m}}, g=\frac{G_{c}}{G_{m}}$.

The formulas obtained by Vanin G.A. [10] have the following form:

$$
\begin{gathered}
E_{1}=E_{c} f+E_{m}(1-f), \\
v_{12}=v_{m}-\frac{g f\left(\chi_{m}+1\right)\left(v_{m}-v_{c}\right)}{(1-f)\left(\chi_{c}-1\right)+g\left(2-f+f \chi_{m}\right)}, \\
E_{2}=\left\{\frac{v_{12}^{2}}{E_{1}}+\frac{\chi_{m}+1}{8 G_{m}}\left[\frac{\chi_{c}-1+2 g}{(1-f)\left(\chi_{c}-1\right)+g\left(2-f+f \chi_{m}\right)}-\frac{2(g-1) f}{1-f+g\left(f+\chi_{m}\right)}\right]\right\}, \\
G_{12}=\frac{1-f+g(f+1)}{f+1+g(1-f)} G_{m}, \\
G_{23}=\frac{1-f+g\left(f+\chi_{m}\right)}{\chi_{m}(f+g(1-f))+1} G_{m},
\end{gathered}
$$

where $\chi_{c}=3-4 v_{c}$.

For transformations of elastic constants $C^{i^{\prime \prime} j^{\prime \prime} k^{\prime \prime}}$ from the coordinate system of reinforcement into the coordinate system of a finite element we use the transformation tensor:

$$
[\alpha]=\left|\begin{array}{ccc}
0 & \cos \alpha & \sin \alpha \\
-1 & 0 & 0 \\
0 & -\sin \alpha & \cos \alpha
\end{array}\right| \text {. }
$$


According to the moment scheme of a finite element, the approximation of displacements takes the form:

$$
u_{k^{\prime}}=\left\{\psi_{i j}\right\}\left\{\omega_{k^{\prime}}\right\}^{T},
$$

where $\quad\left\{\omega_{k^{\prime}}\right\}=\left\{\omega_{k^{\prime}}^{(000)}, \omega_{k^{\prime}}^{(100)}, \omega_{k^{\prime}}^{(010)}, \omega_{k^{\prime}}^{(110)}, \omega_{k^{\prime}}^{(001)}, \omega_{k^{\prime}}^{(101)}, \omega_{k^{\prime}}^{(011)}, \omega_{k^{\prime}}^{(111)}\right\}$ the vector of coefficients of decomposition, $\left\{\psi_{i j}\right\}=\left\{1, x_{1}, x_{2}, x_{1} x_{2}, x_{3}, x_{1} x_{3}, x_{2} x_{3}, x_{1} x_{2} x_{3}\right\}-$ vector of power function.

Deformation tensor we expand by power functions:

$$
\varepsilon_{i j}=\left\{\psi_{i j}\right\}\left\{e_{i j}\right\},
$$

The decomposition coefficients $e_{i j}$ can be written through the displacement approximation coefficients $\omega_{k^{\prime}}^{(p q r)}$ as follows:

$$
\left\{e_{i j}\right\}=\left[F_{i j}^{k^{\prime}}\right]\left\{\omega_{k^{\prime}}\right\} .
$$

Considering the dependence (10), the variation of the elastic energy of deformation (2) will take the form:

$$
\delta W=\iiint_{V}\left\{\delta e_{i j}\right\}^{T}\left\{\psi_{i j}\right\}^{T}\left[C^{i j k l}\right]\left\{\psi_{k l}\right\}\left\{e_{k l}\right\} d V .
$$

The coordinates of an arbitrary point of construction can be represented by the coordinates of the nodes of the finite element and the shape function:

$$
z_{m^{\prime}}=\sum_{L=1}^{8} \tilde{N}_{L}\left(x_{1}, x_{2}, x_{3}\right) z_{m^{\prime}}^{L}
$$

where $z_{k^{\prime}}^{L}$ - the $k^{\prime}$-th coordinate of the $L$-th node in the basic coordinate system (Fig. 1), $k^{\prime}=1,2,3, L=1,2, \ldots, 8, \tilde{N}_{L}\left(x_{1}, x_{2}, x_{3}\right)$ - the form functions of the $L$-th node, which for an infinite finite element will look like: 
for nodes $1-4$ :

$$
\begin{aligned}
& \tilde{N}_{1}\left(x_{1}, x_{2}, x_{3}\right)=-\frac{1}{4}\left(1-x_{1}\right)\left(1-x_{2}\right) \frac{2 x_{3}}{1-x_{3}}, \\
& \tilde{N}_{2}\left(x_{1}, x_{2}, x_{3}\right)=-\frac{1}{4}\left(1+x_{1}\right)\left(1-x_{2}\right) \frac{2 x_{3}}{1-x_{3}}, \\
& \tilde{N}_{3}\left(x_{1}, x_{2}, x_{3}\right)=-\frac{1}{4}\left(1-x_{1}\right)\left(1+x_{2}\right) \frac{2 x_{3}}{1-x_{3}}, \\
& \tilde{N}_{4}\left(x_{1}, x_{2}, x_{3}\right)=-\frac{1}{4}\left(1+x_{1}\right)\left(1+x_{2}\right) \frac{2 x_{3}}{1-x_{3}},
\end{aligned}
$$

for nodes 5-8:

$$
\begin{aligned}
& \tilde{N}_{5}\left(x_{1}, x_{2}, x_{3}\right)=\frac{1}{4}\left(1-x_{1}\right)\left(1-x_{2}\right)\left(1+\frac{2 x_{3}}{1-x_{3}}\right), \\
& \tilde{N}_{6}\left(x_{1}, x_{2}, x_{3}\right)=\frac{1}{4}\left(1+x_{1}\right)\left(1-x_{2}\right)\left(1+\frac{2 x_{3}}{1-x_{3}}\right), \\
& \tilde{N}_{7}\left(x_{1}, x_{2}, x_{3}\right)=\frac{1}{4}\left(1-x_{1}\right)\left(1+x_{2}\right)\left(1+\frac{2 x_{3}}{1-x_{3}}\right), \\
& \tilde{N}_{8}\left(x_{1}, x_{2}, x_{3}\right)=\frac{1}{4}\left(1+x_{1}\right)\left(1+x_{2}\right)\left(1+\frac{2 x_{3}}{1-x_{3}}\right),
\end{aligned}
$$

where $x_{i}^{L}-i$-th coordinate of the $L$-th node in the coordinate system of finite element; $i=1,2,3, L=1,2, \ldots, 8$.

According to the rules of the moment scheme, finally for deformations (10) we will have:

$$
\begin{gathered}
\varepsilon_{11}=e_{11}^{(000)}+e_{11}^{(010)} \psi^{(010)}+e_{11}^{(001)} \psi^{(001)}+e_{11}^{(011)} \psi^{(011)}, \\
\varepsilon_{22}=e_{22}^{(000)}+e_{22}^{(100)} \psi^{(100)}+e_{22}^{(001)} \psi^{(001)}+e_{22}^{(101)} \psi^{(101)}, \\
\varepsilon_{33}=e_{33}^{(000)}+e_{33}^{(100)} \psi^{(100)}+e_{33}^{(010)} \psi^{(010)}+e_{33}^{(110)} \psi^{(110)}, \\
\varepsilon_{12}=e_{12}^{(000)}+e_{12}^{(001)} \psi^{(001)}, \\
\varepsilon_{13}=e_{13}^{(000)}+e_{13}^{(010)} \psi^{(010)}, \\
\varepsilon_{23}=e_{23}^{(000)}+e_{23}^{(100)} \psi^{(100)} .
\end{gathered}
$$


Here, the coefficients of decomposition of deformations will take the form:

$$
\begin{gathered}
e_{11}^{(p q r)}=\sum_{\mu v \eta}^{p q r} \omega_{k^{\prime}}^{(\mu+1 v \eta)} f_{(p+1-\mu q-v r-\eta)}^{k^{\prime}}, \\
e_{22}^{(p q r)}=\sum_{\mu v \eta}^{p q r} \omega_{k^{\prime}}^{(\mu \nu+1 \eta)} f_{(p-\mu q+1-v r-\eta)}^{k^{\prime}}, \\
e_{33}^{(p q r)}=\sum_{\mu v \eta}^{p q r} \omega_{k^{\prime}}^{(\mu v \eta+1)} f_{(p-\mu q-v r+1-\eta)}^{k^{\prime}}, \\
e_{12}^{(p q r)}=\frac{1}{2} \sum_{\mu v \eta}^{p q r}\left(\omega_{k^{\prime}}^{(\mu v v+1 \eta)} f_{(p+1-\mu q-v r-\eta)}^{k^{\prime}}+\omega_{k^{\prime}}^{(\mu+1 v \eta)} f_{(p-\mu q+1-v r-\eta)}^{k^{\prime}}\right) \\
e_{13}^{(p q r)}=\frac{1}{2} \sum_{\mu v \eta}^{p q r}\left(\omega_{k^{\prime}}^{(\mu v \eta+1)} f_{(p+1-\mu q-v r-\eta)}^{k^{\prime}}+\omega_{k^{\prime}}^{(\mu+1 v \eta)} f_{(p-\mu q-v r+1-\eta)}^{k^{\prime}}\right) \\
e_{23}^{(p q r)}=\frac{1}{2} \sum_{\mu v \eta}^{p q r}\left(\omega_{k^{\prime}}^{(\mu \nu v+1 \eta)} f_{(p-\mu q+1-v r-\eta)}^{k^{\prime}}+\omega_{k^{\prime}}^{(\mu \nu+1 \eta)} f_{(p-\mu q-v r+1-\eta)}^{k^{\prime}}\right)
\end{gathered}
$$

where

$$
f_{(\mu v v)}^{k^{\prime}}=\left.\frac{\partial^{\mu+v+\eta} z_{k^{\prime}}}{\left(\partial x_{1}\right)^{\mu}\left(\partial x_{2}\right)^{v}\left(\partial x_{3}\right)^{\eta}}\right|_{x_{1}=x_{2}=x_{3}=0}
$$

Considering these decomposition and relations (10) and (11), the variation of the energy of elastic deformation is written as follows:

$$
\delta W=\iiint_{V}\left\{\delta \omega_{k^{\prime}}\right\}^{T}\left[F_{i j}^{k^{\prime}}\right]^{T}\left\{\psi_{i j}\right\}^{T}\left[C^{i j k l}\right]\left\{\psi_{k l}\right\}\left[F_{k l}^{m^{\prime}}\right]\left\{\omega_{m^{\prime}}\right\} d V
$$

or

$$
\delta W=\left\{\delta \omega_{k^{\prime}}\right\}^{T}\left[F_{i j}^{k^{\prime}}\right]^{T}\left[H^{i j k l}\right]\left[F_{k l}^{m^{\prime}}\right]\left\{\omega_{m^{\prime}}\right\}
$$


where $\left[H^{i j k l}\right]=\int_{-1}^{1} \int_{-1}^{1} \int_{-1}^{1}\left\{\psi_{i j}\right\}^{T}\left[C^{i j k l}\right]\left\{\psi_{k l}\right\} \sqrt{g} d x_{1} d x_{2} d x_{3}$ - the matrix of elastic constants of fibrous composite material taking into account the metric of infinite finite element.

The displacements vector components in the global coordinate system are:

$$
u_{k^{\prime}}=\left\{N_{L}\right\}\left\{u_{k^{\prime}}^{*}\right\}^{T},
$$

where $\left\{u_{k^{\prime}}^{*}\right\}=\left\{u_{k^{\prime}}^{(1)}, u_{k^{\prime}}^{(2)}, u_{k^{\prime}}^{(3)}, u_{k^{\prime}}^{(4)}, u_{k^{\prime}}^{(5)}, u_{k^{\prime}}^{(6)}, u_{k^{\prime}}^{(7)}, u_{k^{\prime}}^{(8)}\right\}$ - the vector of nodes displacement, $\left\{N_{L}\right\}=\left(N_{1}, N_{2}, N_{3}, N_{4}, N_{5}, N_{6}, N_{7}, N_{8}\right)$ - the form function vector of the form:

for nodes $1-4$ :

$$
N_{L}\left(x_{1}, x_{2}, x_{3}\right)=-\frac{1}{4}\left(1+x_{1} x_{1}^{L}\right)\left(1+x_{2} x_{2}^{L}\right) N^{\prime}{ }_{3}\left(x_{3}\right)
$$

for nodes $5-8$ :

$$
N_{L}\left(x_{1}, x_{2}, x_{3}\right)=\frac{1}{4}\left(1+x_{1} x_{1}^{L}\right)\left(1+x_{2} x_{2}^{L}\right) N_{3}^{\prime}\left(x_{3}\right),
$$

here $N_{3}\left(x_{3}\right), \quad N_{3}{ }_{3}\left(x_{3}\right)$ are chosen so that when the coordinate $z_{3^{\prime}}$ tend to infinity the displacements $u_{m^{\prime}}$ would tend to 0 . This imposes certain restrictions on the choice of global coordinates of the finite element, which must correspond to the local coordinates $x_{3}=1-2\left(z_{3^{\prime}}^{M}-z_{3^{\prime}}^{K}\right) /\left(z_{3^{\prime}}-2 z_{3^{\prime}}^{M}+z_{3^{\prime}}^{K}\right)$ (the values of $\mathrm{M}$ and $\mathrm{K}$ are taken in pairs from the values of $M=5,6,7,8$ and $K=1,2,3,4)$; the approximation of displacements is expanded in a series along this coordinate $u_{m^{\prime}}=a_{0}\left(x_{1}, x_{2}\right)+a_{1}\left(x_{1}, x_{2}\right) x_{3}+a_{2}\left(x_{1}, x_{2}\right) x_{3}^{2}+\ldots$ on condition $u_{m^{\prime}} \rightarrow 0$, if $z_{3^{\prime}} \rightarrow \infty$.

From relations (9) and (21), we define the elements of the matrix $[A]$ so that the equality will be hold:

$$
\left\{\omega_{k^{\prime}}\right\}=[A]^{T}\left\{u_{k^{\prime}}^{L}\right\}
$$


Considering (22) we can write for the variation of the energy of elastic deformation:

$$
\delta W=\left\{\delta u_{k^{\prime}}^{L}\right\}^{T}\left[K^{k^{\prime} m^{\prime}}\right]\left\{u_{m^{\prime}}^{L}\right\}
$$

The last relation will determine the stiffness matrix of an infinite finite element based on the moment scheme for the fibrous composite material:

$$
\left[K^{k^{\prime} m^{\prime}}\right]=[A]\left[F_{i j}^{k^{\prime}}\right]^{T}\left[H^{i j k l}\right]\left[F_{k l}^{m^{\prime}}\right][A]^{T} .
$$

\section{Results}

The described stiffness matrix is implemented in the software package MIRELA $+[11]$. This complex solves the problem of indenting three stamps into a multilayered environment. Two cylindrical stamps (2) with a parabolic section are symmetrically relative to the axis of symmetry of a cylindrical stamp (1) with a circular section (Fig. 2).

Initial data: the width of environment is $b=0.5 \mathrm{~m}$, the thickness of each of the three layers is $0.05 \mathrm{~m}$, the total thickness is $t=0.15 \mathrm{~m}$, the length is infinite. The stamps are absolutely rigid. The material of the layers is a fiber composite with a volume fraction of fiber $t$. Elastic constants of the matrix material: the Poisson coefficient is $v_{m}=0.49$; the elastic modulus is $E_{m}=5.28 \mathrm{MPa}$. Elastic constants of the fiber material: the Poisson coefficient is $v_{c}=0.3$, the elastic modulus is $E_{c}=1277.5 \mathrm{MPa}$.

The distance between the stamps is $d=0.4 \mathrm{~m}$. The stamps immersion into the environment to a depth of $0.04 \mathrm{~m}$. The profile of the stamp 1 is described by the equation $-x^{2}+z^{2}=0.2^{2}$, for the stamp $2-x=y^{2}+z^{2}$.

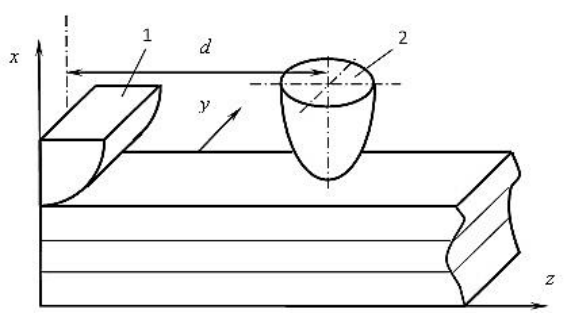

Fig. 2. Contact interaction of stamps with multilayer environment 
Two schemes of fiber reinforcement have been investigated. In the first, $\alpha=90^{\circ}$ for the lower and upper layers, $\alpha=0^{\circ}$ for the middle layer ( $\alpha$ is the angle between the reinforcement direction and the $O y$ axis in the $O y z$ plane). In the second $\alpha=0^{\circ}$ for the lower and upper layers, $\alpha=90^{\circ}$ for the middle layer.

The maximum compressive stresses, depending on the volumetric fiber content in the composite, are shown in Figures 3 and 4.

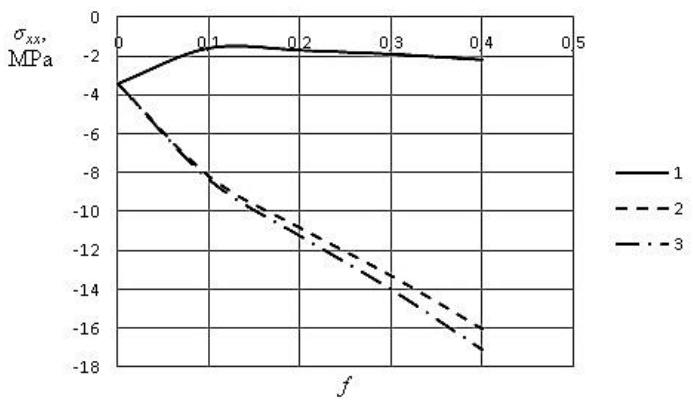

— - the formula (5), - - - - the formula (6), - - - - - the formula (7)

Fig. 3. Maximum compressive stresses

for the first reinforcement scheme

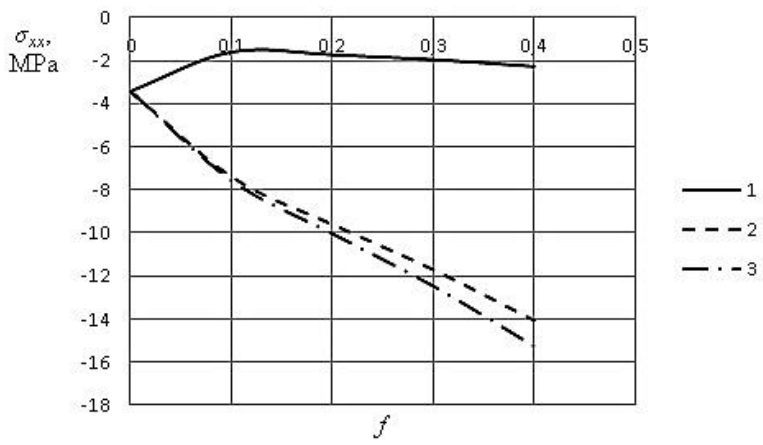

the formula (5), - - - - the formula (6), - - - - - the formula (7)

Fig. 4. Maximum compressive stresses for the second reinforcement scheme 


\section{CONCLUSIONS}

The mixtures rule (5) for determining the effective elastic constants is quite inaccurate, especially when determining the transverse characteristics. Therefore, the magnitudes of the maximum compressive stresses are significantly different from the other two cases. When using formulas (6) and (7) the quality of representation is the same, formulas (7) give 6-8\% higher values of stresses than formulas (6). The maximum stresses increase with increasing $f$ because increasing the content of stiffer fibers increases the rigidity of the entire structure. The first reinforcement scheme gives $10-14 \%$ greater values of maximum compressive stresses than the second.

\section{SUMMARY}

The article investigates the stiffness matrix of infinite hexahedral finite element for fiber composite material based on the moment scheme.

The unique combination of mechanical properties of composite materials allows them to find new applications in various industries: energy, aerospace, construction, transportation, etc. So, one of the branches where they are used is the use of composite materials in the creation of conveyor belts, escalators, conveyor belts, sports and transport road surfaces.

Thus, the presence of composite material, which is heterogeneous in its structure, complicates the mathematical model of construction, which must take into account the presence of a large number of reinforcing elements. In addition, the described construction consists of elements with a specific geometric shape and sizes. These are usually confined strips or very long lengths that can be considered infinite. In the course of operation, such constructions test the load in the local area, which is considerably inferior in sizes to the length of the construction. Therefore, infinite-size modeling is an important problem in solving practical problems.

\section{REFERENCES}

1. K. Edip, V. Sheshov, J. Bojadjieva, A. Demir and H. Ozturk, Development of infinite elements for simulation of unbounded media, Building materials and structures, 61, 2018, 3-13.

2. F. Medina and R.L. Taylor, Finite element techniques for problems of unbounded domains, International journal for numerical methods in engineering, 19(8), 1983, 1209-1226.

3. O.C. Zienkiewicz and R.L. Taylor, The finite element method. Volume 1: The basis, Oxford: Butterworth-Heinemann, 2000, 689. 
4. A. Curnier, A static infinite element, International journal for numerical methods in engineering, 19(10), 1983, 1479-1488.

5. G. Beer, 'Infinite domain' elements in finite element analysis of underground excavations, International journal for numerical and analytical methods in geomechanics, 7, 1983, 1-7.

6. S.V. Choporov, N.I.-V. Manko, O.G. Spytsia and S.M. Grebenyuk, Stiffness matrix of a "semi-infinite" finite element for a weakly compressible material based on the moment scheme, Bulletin of Zaporizhzhia National University, Physical and Mathematical Sciences, 1, 2019, 124-132.

7. S.A. Ambartsumyan, General theory of anisotropic shells, Nauka, Moscow, 1974, 448.

8. D.M. Karpinos, Composite materials. Handbook (D.M. Karpinos ed.), Naukova Dumka, Kiev, 1985, 592.

9. D.S. Abolin'sh, Compliance tensor for an elastic material reinforced in one direction, Polymer mechanics, 4, 1965, 52-59.

10.G.A. Van Fo Fy, Elastic constants and stressed state of glass-fiber ribbon, Polymer mechanics, 4, 1966, 593-602.

11.S.M. Grebenyuk, S.I. Homenyuk and M.I. Klymenko, The stressstrained state of the space constructions on the basis of homogenization of the fiber composites: Monograph. Kherson: Publishing house "Helvetica", 2019, 350.

\section{Information about the authors: Manko N. I.-V.,}

Lecturer at the Department of Software Engineering, Zaporizhzhia National University 66, Zhukovsky str., Zaporizhzhia, 69600, Ukraine

Spytsia O. G., Ph.D. in Phys. and Math., Associate Professor, Associate Professor at the Department of General Mathematics, Zaporizhzhia National University 66, Zhukovsky str., Zaporizhzhia, 69600, Ukraine 